\title{
Contribution of electronic excitation to the structural evolution of ultrafast laser-irradiated tungsten nanofilms
}

\author{
Samuel T. Murphy, ${ }^{1, *}$ Yvelin Giret, ${ }^{1}$ Szymon L. Daraszewicz, ${ }^{1}$ Anthony C. Lim, ${ }^{1,2}$ Alexander L. Shluger, ${ }^{1}$ \\ Katsumi Tanimura, ${ }^{2}$ and Dorothy M. Duffy ${ }^{1}$ \\ ${ }^{1}$ Department of Physics and Astronomy, University College London, Gower Street, WC1E 6BT, London, United Kingdom \\ ${ }^{2}$ The Institute of Scientific and Industrial Research (ISIR), Osaka University, Mihogaoka 8-1, Ibaraki, Osaka, 567-0047, Japan
}

(Received 6 November 2015; revised manuscript received 19 January 2016; published 9 March 2016)

\begin{abstract}
The redistribution of the electron density in a material during laser irradiation can have a significant impact on its structural dynamics. This electronic excitation can be incorporated into two temperature molecular dynamics (2T-MD) simulations through the use of electronic temperature dependent potentials. Here, we study the structural dynamics of laser irradiated tungsten nanofilms using 2T-MD simulations with an electronic temperature dependent potential and compare the results to equivalent simulations that employ a ground-state interatomic potential. Electronic excitation leads to an expansion of the crystal and a decrease in the melting point of tungsten. During laser irradiation these factors ensure that the threshold fluences to the different melting regimes are reduced. Furthermore, both heterogenous and homogeneous melting are predicted to occur more rapidly due to excitation and oscillations in the film thickness will be accentuated.
\end{abstract}

DOI: 10.1103/PhysRevB.93.104105

\section{INTRODUCTION}

During laser irradiation of metals, energy is transferred to the electrons promoting them from their ground state into some excited state. Electron-electron collisions result in the electronic energies adopting a Fermi-Dirac distribution with a well defined electronic temperature $T_{e}$. Energy is then transferred to the ions, via electron-phonon coupling, leading to an increase in the ionic temperature $T_{i}$. If the energy transferred to the ions is sufficient to raise $T_{i}$ above the melting temperature, the material will undergo thermal melting $[1,2]$. For a number of materials, ultrafast laser irradiation resulted in the sample melting on timescales shorter than that required for the electron-phonon coupling to drive the lattice temperature above the melting point [3-6]. This type of melting is often termed nonthermal melting and it was attributed to the strong modification of the interatomic forces owing to laser-induced excitation of a large fraction (10\% or more) of the valence electrons, which results in coherent atomic motions. Evidence for modification of the interatomic potential was provided by Garl et al. [7] by the generation of coherent optical phonons in bismuth. Further support for this hypothesis comes from density functional theory (DFT) molecular dynamics (MD) simulations that showed how high electronic temperatures could lead to disordering of Si [8]. More recent simulations have shown that combining nonthermal effects and electronphonon coupling leads to a reduced excitation threshold to disordering compared to Born-Oppenheimer MD simulations [9]. Melting is not the only lattice response to excitation, a number of solid-solid phase transformations have been either observed or predicted to occur during laser irrdiation [10-18].

Understanding how modifications to the potential energy surface contribute to the structural evolution of a material is a prerequisite for achieving control over these complex processes and the development of new materials with enhanced

*samuel.murphy@ucl.ac.uk properties. However, experimentally it is not possible to completely separate the thermal (i.e., phonon) and nonthermal (i.e., electronic excitation) contributions to the structural evolution of a material during laser irradiation. By contrast, atomistic simulation is ideally suited to the task as it allows the study of atomic dynamics and the isolation of individual parameters and their effects.

A complete description of the electron and ion dynamics during irradiation must be quantum mechanical in nature, however, traditional approaches, such as those based on the Born-Oppenheimer approximation, are unable to describe the transfer of energy between ions and electrons [9]. Timedependent density functional theory (TD-DFT) using Ehrenfest dynamics has demonstrated some success in incorporating energy transfer to the electrons due to electronic drag, however, incorporation of electron-phonon coupling requires more complex approaches, such as correlated electron-ion dynamics [19] or surface hopping [20,21]. Such simulations are computationally demanding and, consequently, not applicable to the large systems required to reproduce structural dynamics observed using time resolved electron or x-ray diffraction. Much larger systems, often consisting of millions of atoms, are accessible to classical MD simulations where the ionic positions are evolved in time according to forces evaluated using an empirically derived force field. In their traditional guise, these simulations do not include electronic information due to their treatment of atoms as simple point objects rather than treating ions and electrons independently. Some of this information, such as electronic stopping and electronphonon coupling, can be reintroduced by coupling the MD supercell to an electronic subsystem, where electronic energy is redistributed according to heat diffusion equations, creating a hybrid continuum-atomistic two-temperature molecular dynamics (2T-MD) model. These 2T-MD simulations have been widely applied to the study of laser irradiation [22-26] as well as radiation damage cascades [27-29].

Although the 2T-MD approach includes some of the relevant electronic effects, the majority of previous studies 
have employed an empirical potential fitted to the ground state of the material and, consequently, neglect the fact that the character of the interactions between atoms changes as a result of excitation. There have been a number of attempts to incorporate these nonthermal effects into simulations employing the 2T-MD model through the use of electronic temperature dependent potentials [18,30-34]. In fact, Daraszewicz et al. have shown that by employing $T_{e}$-dependent potentials it is possible to exactly reproduce the experimentally observed Bragg peak evolution of laser irradiated gold nanofilms [23] at high absorbed fluences. An alternative method for representing the effects of electronic excitation on the interactions between atoms was developed by Rosandi et al. who created an excited state potential for Al by exciting an electron into the conduction band in a DFT supercell [35].

The aim of this study is to compare the structural dynamics of laser irradiated tungsten nanofilms using 2T-MD, with a $T_{e}$-dependent potential, to a series of identical simulations in which the potential does not change with $T_{e}$. This allows us to identify the contribution of the change in the character of interactions induced by electronic excitation to the atomic dynamics during ultrafast laser irradiation. In addition, the simulations highlight the effects that may have been neglected in previous simulations, where ground-state potentials have been used, thereby allowing an evaluation of the large body of existing work.

\section{METHODOLOGY}

\section{A. Two temperature molecular dynamics}

The 2T-MD approach is an evolution of the phenomenological two temperature model (TTM) [36] where energy transfer is described using a series of coupled heat diffusion equations. In the 2T-MD approach, the heat diffusion equation representing the ionic subsystem is replaced by a MD supercell, thereby allowing the evolution of the atomic positions to be followed explicitly $[22,27,28]$. The methodology applied here is identical to that used in previous work [18].

Both the TTM and the 2T-MD models assume that the electronic energies rapidly adopt a Fermi-Dirac distribution with a well defined electronic temperature. We note that at high levels of excitation the number of excited states can outnumber the number of filled states distorting the FermiDirac distribution, however, this has not been considered here. Energy diffusion through the electronic subsystem can be represented using the heat diffusion equation, i.e.,

$$
C_{e}\left(T_{e}\right) \frac{\partial T_{e}}{\partial t}=\nabla \cdot\left(\kappa_{e} \nabla T_{e}\right)-G\left(T_{e}\right)\left(T_{e}-T_{i}\right)+S(z, t),
$$

where $C_{e}\left(T_{e}\right)$ is the electronic specific heat, $G\left(T_{e}\right)$ is the electron-phonon coupling, $\kappa_{e}$ is the electronic heat conductivity, and $T_{i}$ is the local ionic temperature. $S(z, t)$ is a source term that represents energy deposited by the laser. The laser pulse is assumed to be Gaussian in time with an exponentially decreasing amplitude as a function of depth in the film $z$, that is,

$$
S(z, t)=\left(\frac{2 F}{l_{p} t_{p}} \frac{\sqrt{\ln 2}}{\pi}\right) e^{-4 \ln 2\left(t-t_{0}\right)^{2} / t_{p}^{2}} e^{-z / l_{p}},
$$

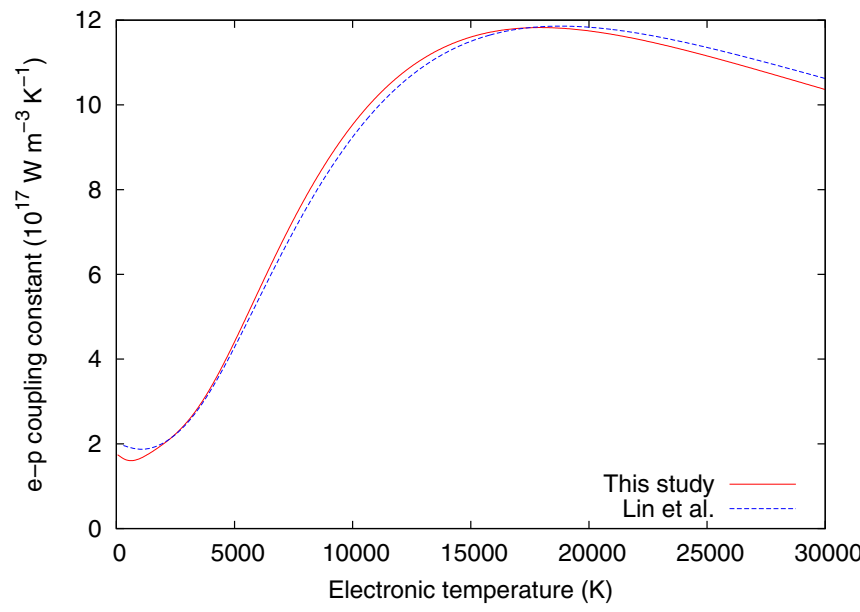

FIG. 1. Electron-phonon coupling for W calculated here compared with the previous work of Lin et al. [39].

where $F$ is the absorbed fluence, $l_{p}$ is the optical penetration depth of the sample at the wavelength of the pulse, $t_{p}$ is the duration of the pulse at the full width half maximum (FWHM), and $t_{0}$ is the time zero corresponding to the maximum of the laser pulse on the sample surface [37]. If the thickness of the target sample is similar to the penetration depth of the laser, the sample will become homogeneously excited and the $\kappa_{e}$ and $z$ dependencies in equations 1 and 2 disappear.

The transfer of energy between the electrons and the lattice is dictated by the electron-phonon coupling strength $G\left(T_{e}\right)$, as shown in equation 1 . The electron-phonon coupling under equilibrium conditions was taken to be $G_{0}=1.65 \times$ $10^{17} \mathrm{~W} \mathrm{~m}^{-3} \mathrm{~K}^{-1}$ as determined by Daraszewicz et al. [38] using DFT. Extrapolation to higher electronic temperatures was done using the methodology of Lin et al. [39]. The density of states required for this extrapolation was calculated using ABINIT [40,41], with the local density approximation, normconserving pseudopotentials and a fine Monkhorst-Pack $k$-point grid. The $T_{e}$-dependent electron-phonon coupling is presented in Fig. 1 and shows good agreement with the work of Lin et al. [39]. This derivation neglects the contribution of electron-phonon momentum exchange and therefore neglects the effect of lattice temperature on energy transfer $[42,43]$, which has been shown to increase by up to two orders of magnitude [44,45]. However, it has been shown that this $T_{e}$-dependent electron-phonon coupling is capable of closely reproducing the temperature profiles for tungsten single crystals subject to irradiation by a 90 fs laser pulse [38].

Similarly, the electronic specific heat exhibits a dependency on $T_{e}$ and this was also calculated using finite temperature DFT. For low electronic temperatures, such as those encountered here, our calculated electronic specific heats are in excellent agreement with Lin et al. and more recent DFT studies [46-48].

The traditional equations of motion governing the evolution of atoms in the MD supercell are modified, such that

$$
m_{i} \frac{\partial \mathbf{v}_{i}}{\partial t}=\mathbf{F}_{i}+\tilde{\mathbf{F}}_{i}\left(T_{t}\right)
$$


where $\mathbf{v}_{i}$ is the velocity of an atom with mass, $m_{i} . \mathbf{F}_{i}$ is the classical force acting on the atom calculated using an empirical potential (described below) and $\tilde{\mathbf{F}}_{i}$ is the additional driving term, based on a modified Langevin thermostat formulation:

$$
\tilde{\mathbf{F}}_{i}\left(T_{t}\right)=-\gamma \mathbf{v}_{i}+\mathbf{f}_{L}\left(T_{t}\right),
$$

where $\gamma$ represents a frictional drag force, $\mathbf{f}_{L}$ is the stochastic force and $T_{t}$ is the target temperature of the thermostat, which is set equal to the local electronic temperature, thereby allowing the thermostat to mimic the electron-phonon coupling.

\section{B. $T_{e}$-dependent potential}

The $T_{e}$-dependent potential employed here was developed in previous work by fitting to free energy-volume curves calculated using finite temperature DFT [18]. More details of the potential and its derivation can be found in this previous work, however, it is beneficial for the following discussion of the structural dynamics of tungsten to briefly review some of the features of the new potential.

The two key features of interest here are the increase in the equilibrium volume [indicated by black crosses in Fig. 2(a)] with electronic temperature and the decrease in the depths of the potential wells (i.e., the binding energy). The increase in the equilibrium volume implies that the interaction between $\mathrm{W}$ atoms becomes more repulsive as $T_{e}$ increases. Additionally, the decrease in the well depths as $T_{e}$ increases implies that the melting temperature of tungsten will decrease due to electronic excitation. Figure 2(b) clearly shows how the melting point (calculated using the moving interface method [49]) decreases as the electronic temperature decreases. At $T_{e}=30000 \mathrm{~K}$, the tungsten supercell was predicted to melt even when the ionic temperature was set to $1 \mathrm{~K}$. A similar observation was made for Si by Shokeen and Schelling [32] implying that the decrease in melting temperature due to electronic excitation may be general for different classes of materials. In addition to correctly reproducing the changes in the lattice parameter as a function of electronic temperature predicted using finite temperature DFT, the $T_{e}$-dependent potential is able to correctly reproduce changes in the dynamical stabilities of the bcc and fcc phases of $\mathrm{W}$ due to excitation [18].

\section{Computational procedure}

Laser irradiation simulations were performed using a local version of the DL-POLY (4.01) code [50] on $\mathrm{a} \approx 190$ - $\AA$-thick tungsten thin film. The film was created by taking $30 \times 30 \times$ 60 repetitions of the bcc unit cell and contained 108000 atoms. The simulation supercell was periodic in $x$ and $y$ and the supercell length in $z$ was doubled to allow for any possible expansion during laser irradiation, with the final supercell lattice parameters of $96.18 \times 96.18 \times 384.72 \AA$. The cell boundaries remain fixed during the simulation as the area of the laser spot is greater than the simulation cell and the excited atoms surrounding the simulation cell impart a sufficient pressure to prohibit expansion in the lateral directions.

The simulation cell was then subdivided into a grid of $9 \times$ $9 \times 36$ cubic local temperature voxels measuring $\approx 11 \AA$. Each voxel was connected to an electronic voxel in the continuum cell. In order to avoid electronic temperature leaking into the

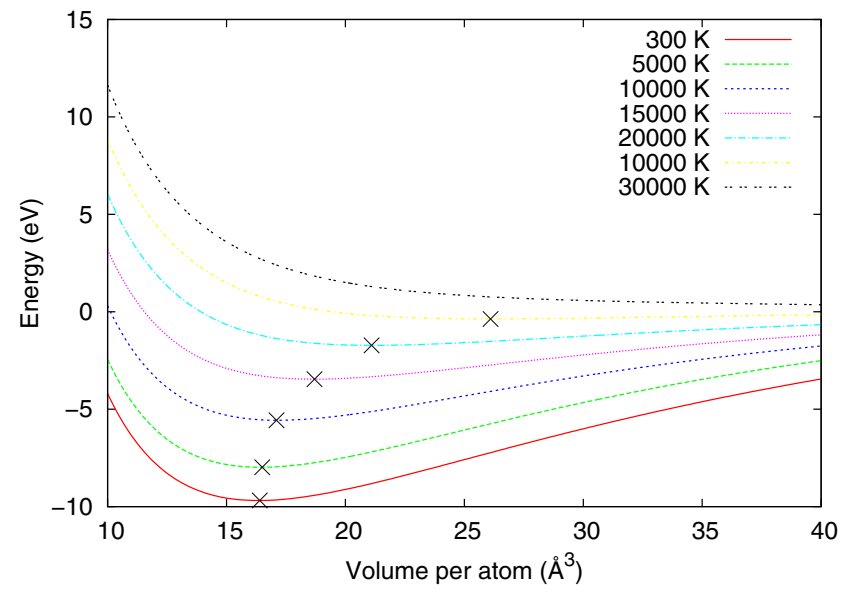

(a)Energy-volume curves

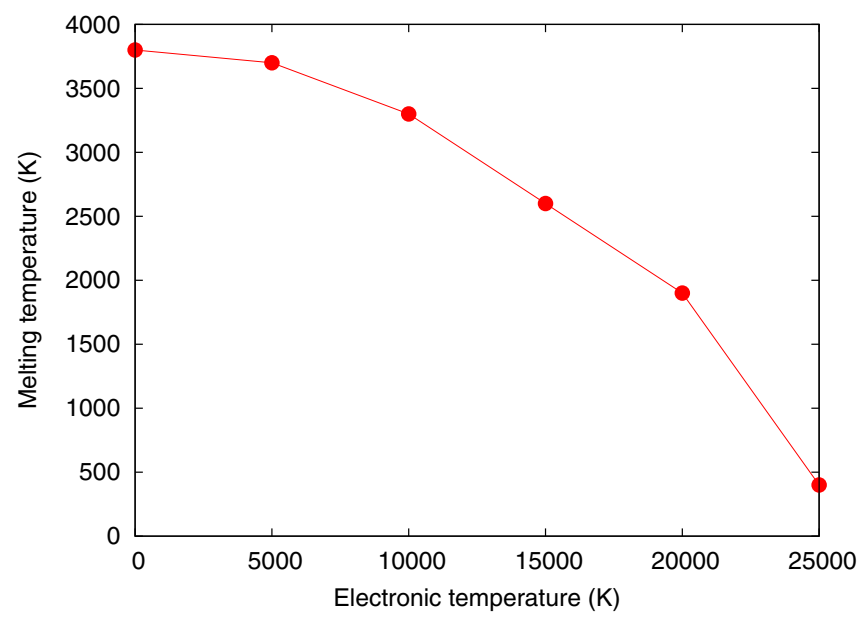

(b)Melting temperature

FIG. 2. Energy volume curves for bcc $\mathrm{W}$ at a series of electronic temperatures (a). Black crosses indicate the minimum in the energy volume curves. The decrease in the well depths of the potentials indicates that the melting point will decrease as $T_{e}$ increases as shown in (b). These plots are based on data from Murphy et al. [18].

vacuum region, only atom containing voxels were allowed to transfer energy between the ionic and electronic subsystems. As the film expanded voxels were awakened when the number of atoms in that voxel exceeded a threshold value, taken here as 20. Similarly, when the number of atoms dropped below the same threshold the voxels were closed. For more details of the method, refer to Daraszewicz et al. [23].

The ionic simulation supercell was initially relaxed under constant volume conditions using a Nose-Hoover thermostat at $300 \mathrm{~K}$ for $6 \mathrm{ps}$ with a $1 \mathrm{fs}$ time step for the dynamics. Once equilibration was complete, the ionic system was connected to the continuum system and the initial electronic temperature set to $300 \mathrm{~K}$. A laser pulse lasting $76 \mathrm{fs}$ at the FWHM with a penetration depth of $150 \AA$ was then initiated and the system was subsequently allowed to evolve for up to $300 \mathrm{ps}$ again with a $1 \mathrm{fs}$ time step for low fluences $\left(\leqslant 35 \mathrm{~mJ} \mathrm{~cm}^{-2}\right)$ and $0.2 \mathrm{fs}$ for higher fluences. Fluences ranging from $10 \mathrm{~mJ} \mathrm{~cm}^{-2}$ up to a high fluence of $50 \mathrm{~mJ} \mathrm{~cm}{ }^{-2}$ were studied, in order to ensure all the different melting regimes were examined. 


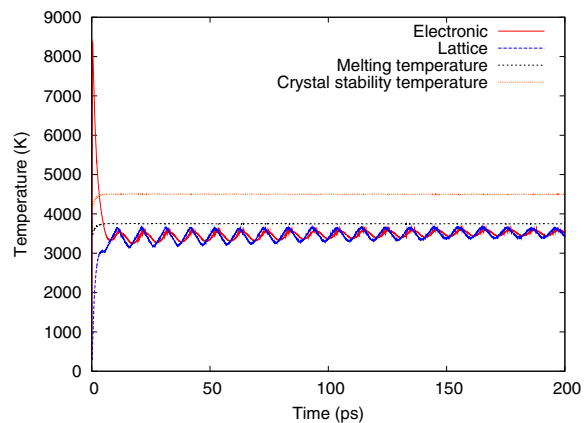

(a) $14 \mathrm{~mJ} \mathrm{~cm}^{-2}$

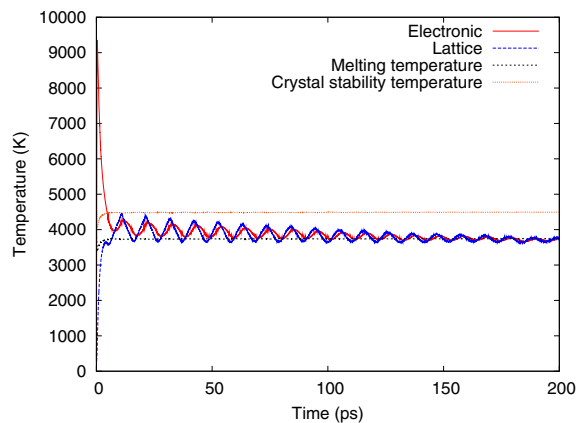

(b) $18 \mathrm{~mJ} \mathrm{~cm}^{-2}$

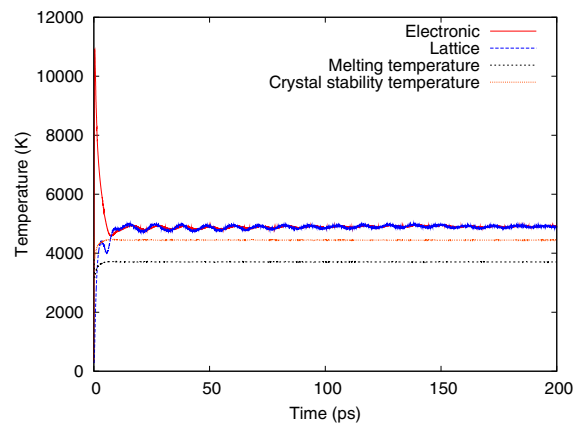

(c) $25 \mathrm{~mJ} \mathrm{~cm}^{-2}$

FIG. 3. Evolution of $T_{i}$ and $T_{e}$ as a function of time for (a) $F=14 \mathrm{~mJ} \mathrm{~cm}^{-2}$ (nonmelting regime), (b) $F=18 \mathrm{~mJ} \mathrm{~cm}^{-2}$ (heterogeneous melting regime), and (c) $F=25 \mathrm{~mJ} \mathrm{~cm}^{-2}$ (homogeneous melting regime). Also included in the plots are the $T_{e}$-dependent melting and crystal stability temperatures where $T_{m}$ is calculated by fitting a spline function to our previously published data on the melting point of $\mathrm{W}$ as a function of $T_{e}[18]$.

\section{RESULTS AND DISCUSSION}

\section{A. Melting dynamics}

A material's structural response to ultrafast laser irradiation is believed to have two distinct stages: a nonequilibrium stage where the electronic temperature is significantly greater than the ionic temperature and a second stage where the ionic and electronic systems are in equilibrium. Figure 3 shows the calculated time evolution of the ionic and electronic temperatures during laser irradiation, using the $T_{e}$-dependent potential for three fluences. The plots show that the time taken for the films to reach equilibrium is less than $10 \mathrm{ps}$ in all cases.

The high electronic temperatures present in the very early stages will redistribute the electron density, leading to a significant change in the interatomic interactions that dominate the early stage atomic motion. When the ground-state potential is used for the simulations, the modifications to the interactions are neglected, so the atomic motion is dominated by the transfer of energy from the electronic system alone. However, when the $T_{e}$-dependent potential is used, the increase in $T_{e}$ reduces the attraction between ions and the melting point is decreased (indicated in Fig. 3). During the initial nonequilibrium regime the increase in the lattice temperature is driven by electron-phonon coupling and the temperatures changes are similar for both the $T_{e}$-dependent and ground-state potentials.

As the energy is transferred to the ions via electron-phonon coupling, and $T_{e}$ decreases, the influence of the excitation is reduced. We note that as we are employing a closed system, the energy is not removed and the temperatures remain high for the duration of the simulation. Consequently, the potential does not return to the ground state, although as the equilibrated electronic temperatures are low the modification to the potential is not significant.

The structural evolution of the thin film following laser irradiation can be estimated from the temperature of the lattice relative to the melting and crystal stability temperatures. This can be expressed as the degree of overheating represented by $T_{i} / T_{m}$ [51]. The degree of overheating after equilibration as a function of the absorbed fluence is given in Fig. 4.

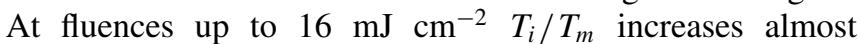
linearly with absorbed fluence and remains below unity. In this region, we observe no melting of the sample as the lattice temperature remains below the $T_{e}$-dependent melting temperature. For fluences in the range $17-20 \mathrm{~mJ} \mathrm{~cm}^{-2}$, the degree of overheating is predicted to be almost constant at unity. This indicates the existence of a latent heat for melting as increased energy provided by the laser does not lead to increased lattice temperatures. A similar observation has been made in simulations of laser melting of silicon [33].

While $T_{i} / T_{m}=1$ the simulations predict a heterogeneous melting process where the melt fronts propagate from the films' surfaces. In these low fluence regimes, the degree of overheating is predicted to be almost identical for the $T_{e}$-dependent and ground-state potentials due to the relatively small changes to the interactions between atoms at these relatively low electronic temperatures. While it appears that

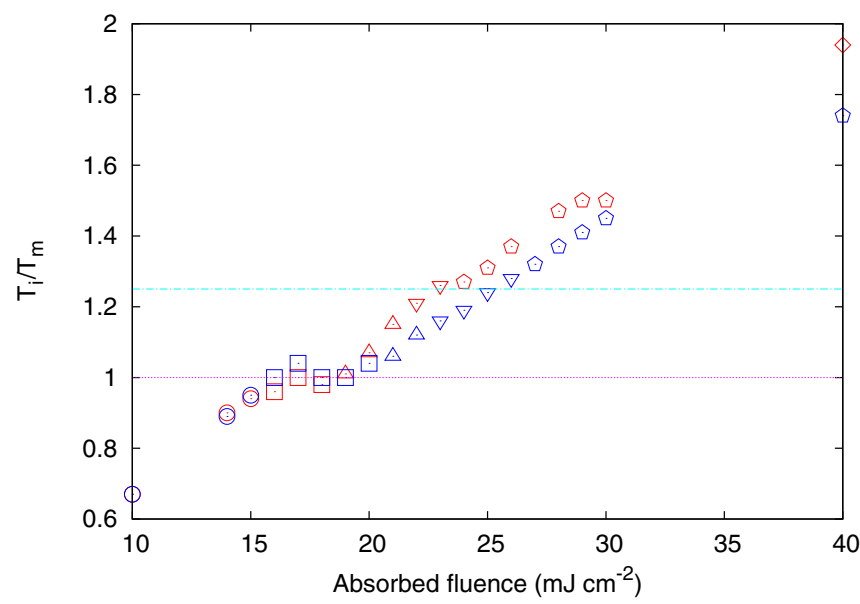

FIG. 4. Degree of overheating $\left(T_{i} / T_{m}\right)$ in the $\mathrm{W}$ thin film as a function of the absorbed fluence calculated using the $T_{e}$-dependent potential (red) and the ground-state potential (blue). The shape of the points represent different structural evolutions observed in the simulations: circles represent the nonmelting regime, squares correspond to the heterogeneous melting regime, triangles represent a mixed heterogeneous-homogenous region, upside down triangles denote purely homogeneous melting and the hexagons indicate homogeneous melting with void formation and diamonds indicate a break up of the film. 


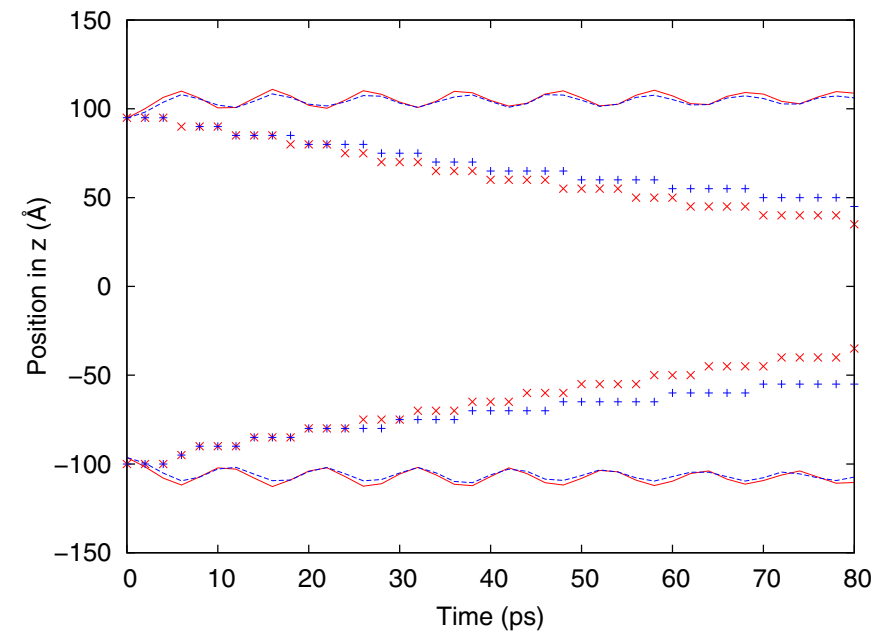

FIG. 5. Plot showing the locations of the surfaces and melt fronts for a $\mathrm{W}$ thin film as a function of time after irradiation with a laser pulse of $17 \mathrm{~mJ} \mathrm{~cm}^{-2}$. The red and blue lines correspond to the position of the surfaces using the $T_{e}$-dependent and ground-state potentials, respectively. The red crosses correspond to the position of the melt front for the $T_{e}$-dependent potential simulation and the blue crosses indicate the position of the melt front for the ground-state potential simulations.

the degree of overheating does not reveal any substantial differences, the predicted structural evolutions are significantly modified. Firstly, the introduction of the repulsive forces when the $T_{e}$-dependent potential drives expansion of the film, as will be discussed later. Also significant are the differences in the melt front velocities. The position of the melt fronts originating from the top and bottom of the film as a function of the simulation time for a laser pulse with $F=17 \mathrm{~mJ} \mathrm{~cm}^{-2}$ are presented in Fig. 5. The melt front is defined as the position where the root mean squared displacement (rMSD) in the $x y$ plane exceeds $1 \AA$ (a detailed explanation for this choice is presented in the Appendix). The figure shows that the melt front advances through the sample more rapidly when the $T_{e}$-dependent potential is employed. This difference is not apparent during the very early stages of the simulation, as the increased repulsion of the $T_{e}$-dependent potential drives expansion of the film prior to melting.

At a fluence of $19 \mathrm{~mJ} \mathrm{~cm}^{-2}$ using the $T_{e}$-dependent potential we observe homogeneous nucleation at the center of the film in addition to the heterogeneous nucleation observed at lower fluences. Overlapping of the hetero- and homogeneous regimes in thin films has been observed previously [24]. The homogeneous nucleation is caused by the formation of a low density region at the center of the film due to the rapid expansion after laser irradiation. Homogeneous nucleation is not observed at this fluence with the ground-state potential where the threshold for this type of melting is predicted to be $21 \mathrm{~mJ} \mathrm{~cm}^{-2}$. The overheating for the ground-state potential at the onset of homogeneous nucleation is 1.06 , in good agreement with similar observations of Ivanov and Zhigilei [52], however, the build up of tensile stresses at the center of the film is exacerbated by the increased repulsion due to excitation, particularly in the nonequilibrium early stages. This additional stress, coupled with the decrease in the melting temperature

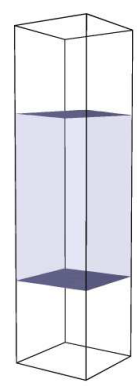

(a) 0 ps



(b)14 ps

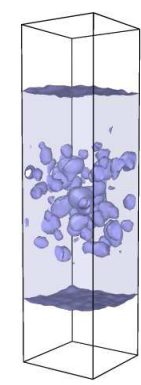

(c) $18 \mathrm{ps}$

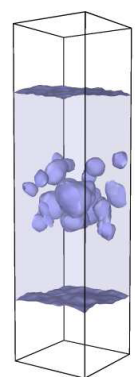

(d)30 ps

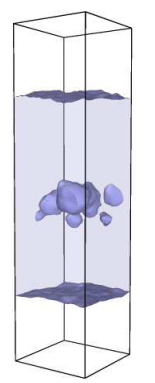

(e) $40 \mathrm{ps}$

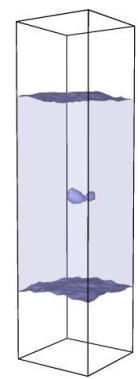

(f) $50 \mathrm{ps}$
FIG. 6. Graphical representation of the appearance of nano voids in $\mathrm{W}$ thin films subjected to a laser pulse delivering $30 \mathrm{~mJ} \mathrm{~cm}{ }^{-2}$. The shaded region represents the film with surfaces clearly visible. As the simulation proceeds, small cavities start to form, which coalesce to form large voids at the center of the film and subsequently close by 50 ps.

due to excitation, results in the degree of overheating required for homogeneous melting being reduced to $1.01 \times T_{m}$.

For a fluence of $22 \mathrm{~mJ} \mathrm{~cm}^{-2}$ with the $T_{e}$-dependent potential and $23 \mathrm{~mJ} \mathrm{~cm}^{-2}$ with the ground-state potential the homogenous melting of the sample occurs so quickly that no heterogeneous nucleation can be identified. From the onset of the mixed heterogeneous-homogenous melting regime the degree of overheating increases almost linearly with the fluence up until the crystal stability temperature is reached. As the threshold to these melting regimes is different, the points for the $T_{e}$-dependent and ground-state potentials are parallel rather than on top of each other, as was the case at lower fluences. Once the degree of overheating reaches the crystal stability limit of $1.25 \times T_{m}$, the sample again melts homogeneously, however, the formation of voids is also observed. These voids form as a result of the tensile stress near the center of the film arising from the initial expansion of the thin film. Similar observations have been made in previous MD [53] and 2T-MD studies [22,52]. At fluences up to $40 \mathrm{~mJ} \mathrm{~cm}^{-2}$ these nanovoids are predicted to form during the initial expansion of the sample. They, subsequently, coalesce to form larger voids, which then shrink until disappearing completely by $50 \mathrm{ps}$, as shown in Fig. 6. As there are no atoms contained within the voids, this process is expected to be mechanically driven [52]. Consequently, it is not surprising that the threshold fluence for creation of these voids is reduced when the atomic interaction becomes more repulsive due to excitation.

When the fluence reaches $40 \mathrm{~mJ} \mathrm{~cm}{ }^{-2}$ the lattice temperature in the $T_{e}$-dependent case reaches almost twice $T_{m}$. The structural evolution of this film is presented in Fig. 7. Upon excitation, the film expands leading to the formation of small nano voids, as discussed above. As before, the voids coalesce, however, the coalescence of these voids leads to the almost complete separation of the top and bottom of the film, splitting it in two. For this fluence the ground-state potential predicts $T_{i} / T_{m}=1.74$, and the structural evolution suggests that the film will remain in a single piece. By contrast, breakup of the nanofilm is predicted to occur for $F=50 \mathrm{~mJ} \mathrm{~cm}{ }^{-2}$ where $T_{i} / T_{m}=2.02$. Again the origin of the different threshold fluences is the increase in repulsion arising from excitation. 



(a) 0 ps (c) 8 ps

(d) $12 \mathrm{ps}$

(e) $16 \mathrm{ps}$
FIG. 7. Structural evolution of the $\mathrm{W}$ nanofilm irradiated with a 76-fs laser pulse with an absorbed fluence of $40 \mathrm{~mJ} \mathrm{~cm}^{-2}$ predicted using the $T_{e}$-dependent potential. Atoms are coloured according to their local density in the range $0.0004-0.525$ atoms per $\AA^{3}$.

We note that Figs. 6 and 7 indicate that the densities across the thin films are not uniform, therefore, the melting point is also not uniform across the film. However, despite this our results indicate that the melting behavior of the film is dictated by the average temperature relative to the melting point at this average temperature (see Figs. 3 and 4).

\section{B. Oscillations in the film thickness}

One of the properties of thin films that can be observed during laser irradiation, using either femtosecond electron or x-ray diffraction, is the film thickness. Plots of the film thickness as a function of time for a range of fluences are presented in Fig. 8. It is evident from this figure that for all fluences considered the average film thickness is greater when the $T_{e}$-dependent potential is used. The increase in the film thickness arises from the increased repulsion associated with the potential [18]. At all but the highest fluences there are clear oscillations in the film's thickness caused by the elastic

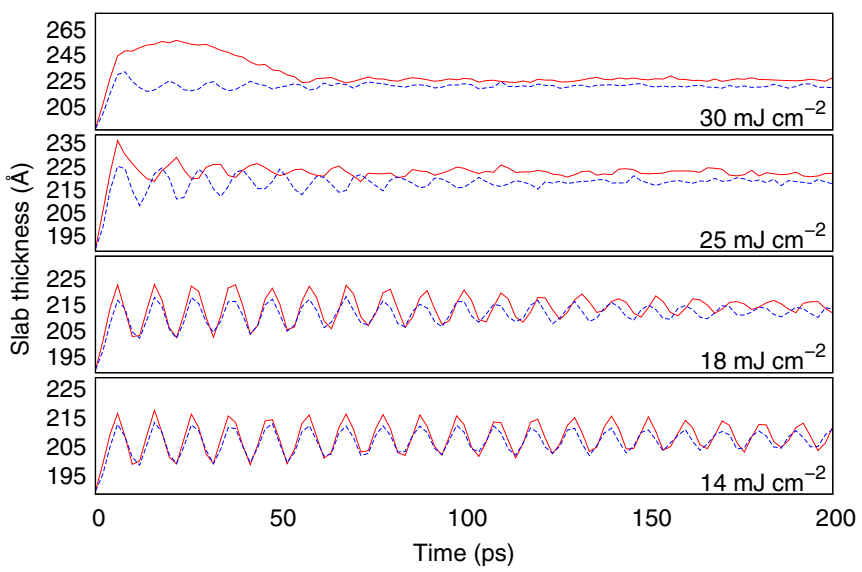

FIG. 8. Thickness of the $\mathrm{W}$ thin films irradiated with a 76-fs laser pulse of different fluences as a function of time. Red lines correspond to results calculated using the $T_{e}$-dependent potential and the blue dashed line represents the data collected using the groundstate potential.

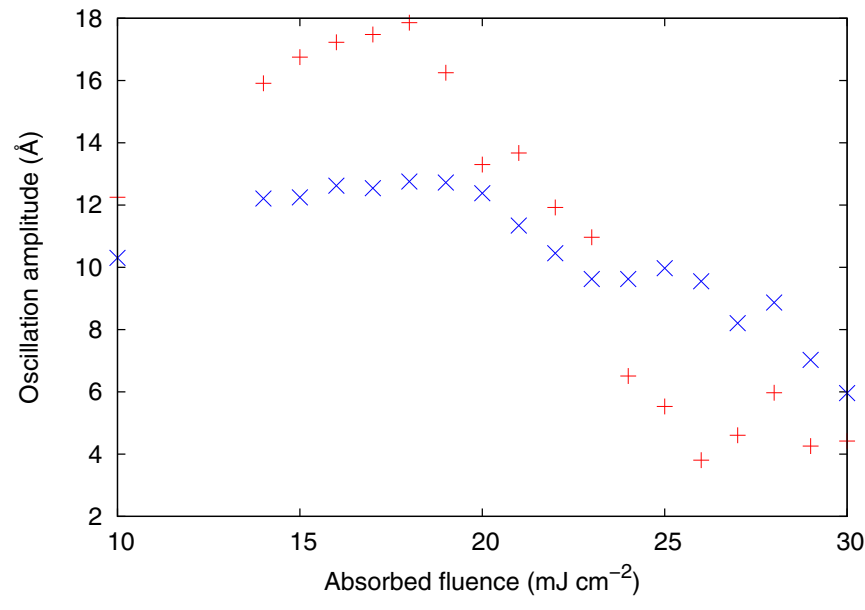

FIG. 9. Amplitude of the oscillation in the thickness (averaged over the first eight periods) of the thin films irradiated with a 76-fs laser pulse of different fluences as a function of time. Red points correspond to results calculated using the $T_{e}$-dependent potential and the blue points represent the data collected using the ground-state potential.

response of the film to the rapid deposition of such a substantial amount of energy. When the $T_{e}$-dependent potential is used, the oscillations in the film thickness are greater than observed when the ground-state potential is used, particularly during the nonequilibrium stage.

As shown in Fig. 9, the amplitude of the oscillations (averaged over the first eight periods) increases with the absorbed fluence irrespective of whether the $T_{e}$-dependent potential was used. Both the amplitude and increase in the amplitude of the oscillations are greater when the $T_{e}$-dependent potential is used. The increase in the amplitude is a result of fluctuations in the electronic temperature illustrated in Fig. 3 causing oscillations in the interatomic potential. This effect becomes more significant as the absorbed fluence increases, thus the amplitude increases more rapidly when electronic excitation is taken into account. At intermediate fluences, the increase in the amplitude of the oscillations ceases as the sample melts, leading to a loss of elasticity. Initially, in the heterogenous regime, only part of the sample melts during the first eight periods and so the effect is small, however, at higher fluences the sample melts completely during this time. The loss of elasticity accompanying the phase transition leads to the dramatic decrease in the amplitude of the oscillations. This decrease in the oscillation amplitude is more dramatic when electronic excitation is considered as the samples melt more rapidly, which results in a more rapid decrease in the average amplitude. At the highest fluences, the initial expansion is predicted to be significantly greater. In particular, at $30 \mathrm{~mJ} \mathrm{~cm}^{-2}$, the film retains a large expansion for a relatively long time period due to the formation of the nanovoids.

Recent experimental observations suggest that the period for these oscillations should be $2 L / v_{c}$, where $L$ is the film's thickness and $v_{c}$ is the speed of sound in the material [54,55]. The data presented here allow us to make atomic scale observations of this behavior. Figure 8 shows that, as the fluence increases the film expands more and so the average 


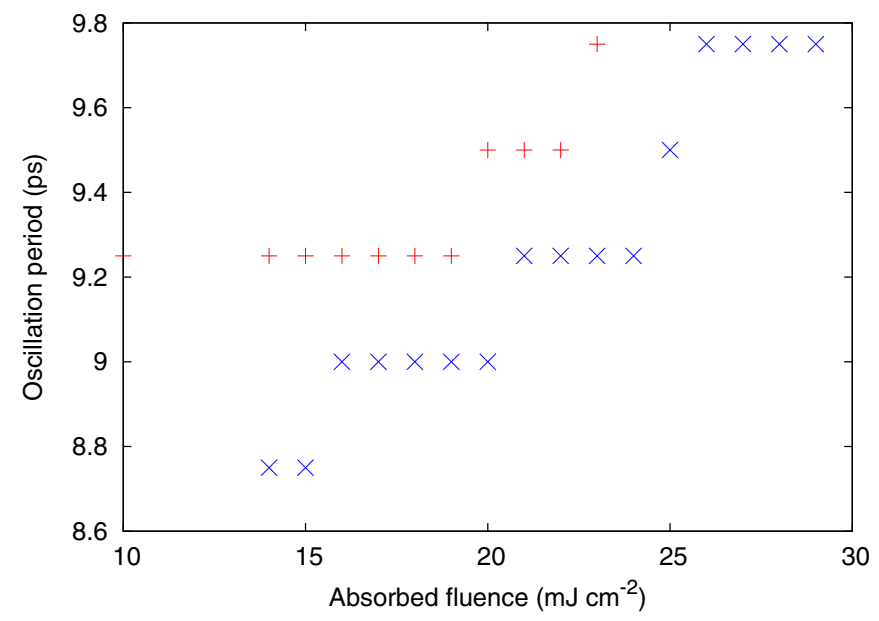

FIG. 10. Predicted period of oscillation for the $\mathrm{W}$ thin films as a function of imparted fluence. The red and blue points denote data calculated using the $T_{e}$-dependent and ground-state potentials, respectively.

thickness is increased. Assuming therefore that $L$ increases with the fluence, it would be expected that the oscillation period would also increase, and that this increase should be greater when the $T_{e}$-dependent potential is used. Figure 10 shows the average period for the first eight oscillations following laser irradiation as a function of the imparted fluence. As expected the oscillation periods are slightly larger when the $T_{e}$-dependent potential is employed. This analysis ignores the influence of changes in the speed of sound in the material due to either changes in the potential or a phase change, however, these effects are expected to be relatively small. Above the threshold for the formation of the nanovoids the oscillation periods show significant variation because the oscillation occurs only in part of the film.

\section{CONCLUSIONS}

The results of 2T-MD simulations of ultrafast laser irradiated $\mathrm{W}$ nanofilms using a $T_{e}$-dependent potential and a ground-state potential have been compared to highlight the important role of electronic excitations in determining the structural evolution of such ultrafast processes. In W, we observe the following effects:

(1) Electronic excitation influences the structural dynamics of materials subjected to laser irradiation even in the low fluence regimes that are typically considered to be in the "thermal" regime. One of the major consequences of electronic excitation is shown to be a decrease in the melting temperature. For melting to occur, the lattice temperature must still exceed this $T_{e}$-dependent melting temperature, hence the structural dynamics observed are similar to the usual "thermal" process. As the melting point has been reduced the amount of energy that must be transferred to the ions via electron-phonon coupling is also decreased, thereby reducing the time required for melting. At very high electronic temperatures, the melting temperature can become very low, therefore melting can occur on very short time scales, i.e., less than a picosecond.

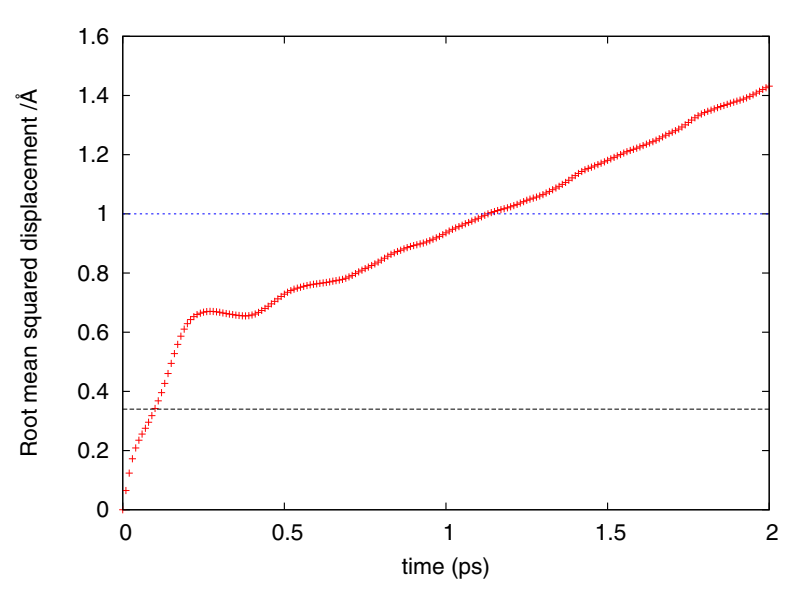

(a) $\operatorname{rMSD}(x y)$

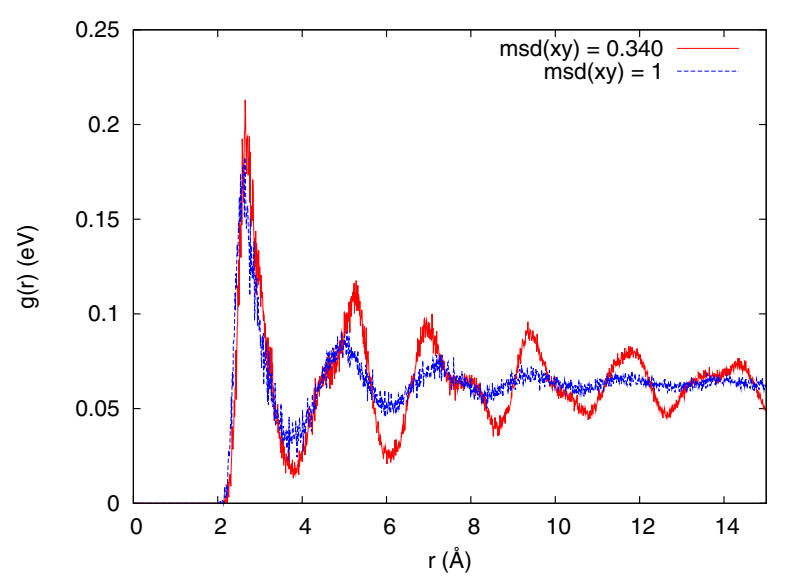

(b)Radial distribution function

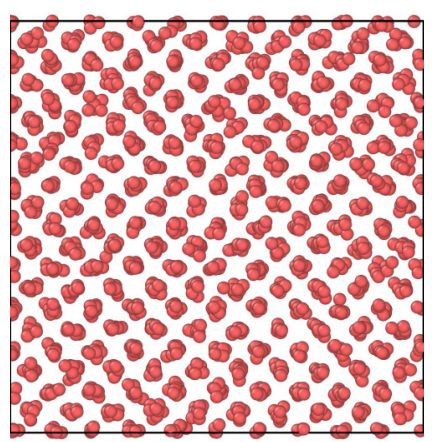

(c) $\mathrm{rMSD}=0.34 \AA$

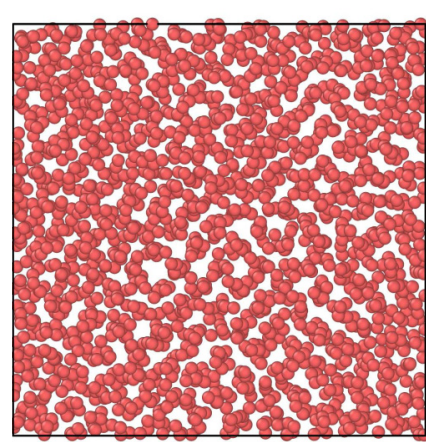

(d) $\mathrm{rMSD}=1.0 \AA$
FIG. 11. Examination of melting in a 2000 atom tungsten supercell at $6000 \mathrm{~K}$. The $\operatorname{rMSD}(x y)$ is presented as a function of time in (a) and the RDF is plotted at the times that the $\operatorname{rMSD}(x y)$ exceeds the Lindemann criteria $(0.34 \AA)$ and $1 \AA$ are shown in (b). The atomic structures at the time when these thresholds are crossed are shown in (c) and (d).

(2) The decrease in $T_{m}$ coupled with increased tensile stresses in the nanofilm due to excitation leads to a decrease in the overheating $\left(T_{i} / T_{m}\right)$ required for the different melting regimes, thereby reducing the threshold fluences. In particular, the overheating required for the onset of homogeneous nucleation is predicted to be $1.01 \times T_{m}$ rather than the $1.06 \times T_{m}$ predicted previously. 
(3) Melt-front velocities in the heterogenous melting regime are predicted to be greater for intermediate fluences. This is due to a combination of a weakening of the bonding interaction between atoms and the decrease in the density resulting from the increased expansion of the film driven by electronic excitation.

(4) Electronic excitation leads to greater expansion of the thin film immediately following laser irradiation. The increased thickness of the film ensures an increase in the oscillation period in agreement with the relation $\left(2 L / v_{c}\right)$. In addition, the amplitude of the oscillations in the film thickness at fluences below the melting threshold are increased due to oscillations in $T_{e}$, which results in oscillations in the strength of the interatomic interaction.

While in this work only $\mathrm{W}$ has been investigated, we speculate that these effects will be general for metals. This prediction is made based on our results for the $s$-type metal gold where the incorporation of nonthermal forces via a $T_{e}$-dependent potential was seen to reduce the time taken for the disappearance of the (200) and (220) Bragg peaks as well as the shape of the free-energy volume curves for metals such as tantalum [56], which are very similar to those observed for W.

In conclusion, the results presented here suggest that the incorporation of the effects of electronic excitation into interatomic potentials does significantly affect the ion dynamics but it does not fundamentally change our understanding of laser induced melting, particularly at lower and intermediate fluences. The different melting processes observed in previous work remain unchanged, however, there are quantitative differences, such as reductions in the thresholds required for the different melting regimes and increased melt front velocities. This demonstrates that the use of $T_{e}$-dependent potentials is essential for quantitative understanding of melting dynamics of materials during laser irradiation.

\section{ACKNOWLEDGMENTS}

STM acknowledges funding from the Leverhulme trust (Grant No. RPG-2013-331). Via our membership of the UK's HPC Materials Chemistry Consortium, which is funded by EPSRC (EP/L000202), this work made use of the facilities of HECToR and ARCHER, the UK's national high-performance computing service, which is funded by the Office of Science and Technology through EPSRC's High End Computing Programme. Additional computational resources were provided by the high performance computer center at UCL.

\section{APPENDIX: DEFINITION OF MELTING}

In many previous studies, differentiation between melted and crystalline material is achieved using the centrosymmetry parameter. Here, however, the rapid oscillations in the film's thickness lead to oscillations in the centrosymmetry parameter that swamp the signal arising from the melting. Therefore we adopt a method based on the Lindemann criterion. The Lindemann criterion states that melting occurs when there is a transformation of harmonic atomic vibrations (phonons) at some temperature into nonlinear oscillations. This was believed to occur when the root mean squared displacement (rMSD) reached a certain fraction of the interatomic spacing, although this exact fraction is ill defined. It should, however, be noted that there are more advanced methods that allow a deep physical interpretation of the equilibrium and nonequilibrium melting independent of numerical model [57].

As was the case for the centrosymmetry parameter, the substantial movement due to the expansion of the film in $z$ leads to oscillations in the rMSD. In order to negate the effect of oscillations in the film thickness we only consider diffusion in the $x y$ plane. The rationale for this is that in a crystalline system atoms will be unable to diffuse in the $x y$ plane, however, once melted they will be free to do so.

The Lindemann criterion states that, if the rMSD exceeds $15 \%$ of the nearest neighbor bond distance, then a material has melted [58]. Here, we examine this particular threshold for the definition of melting by performing MD simulations of a small 2000 bcc tungsten supercell with the ground-state potential and a timestep of 0.01 fs. The simulation was performed under NVT conditions with the Nose-Hoover thermostat and a target temperature of $6000 \mathrm{~K}$. We then follow the rMSD as a function of time and plot the radial distribution function (RDF) at the time when the MSD crosses the Lindemann threshold in Fig. 11.

Figure 11 shows that at the point where the $\operatorname{rMSD}(x y)$ crosses the Lindemann threshold of $0.34 \AA$ (15\% of the $x y$ distance to a nearest neighbor at $2.83 \AA$ ) the RDF still displays some characteristics that would be expected of a solid, such as shoulders on the peaks at $4.5 \AA$. However, when the $\mathrm{rMSD}=1 \AA$ the RDF more closely represents a liquid state. This is confirmed by examining the structure of the supercell at these times, see Figs. 11(c) and 11(d). Therefore we suggest that for identifying a region that has melted as opposed to one that will melt it is better to wait for the $\operatorname{rMSD}(x y)$ to exceed 1 A.
[1] K. H. Bennemann, Ultrafast dynamics in solids, J. Phys.: Condens. Matter 16, R995 (2004).

[2] Y. Giret, N. Naruse, S. L. Daraszewicz, Y. Murooka, J. Yang, D. M. Duffy, A. L. Shluger, and K. Tanimura, Determination of transient atomic structure of laser-excited materials from time-resolved diffraction data, Appl. Phys. Lett. 103, 253107 (2013).

[3] H. W. K. Tom, G. D. Aumiller, and C. H. Brito-Cruz, TimeResolved Study of Laser-Induced Disorder of Si Surfaces, Phys. Rev. Lett. 60, 1438 (1988).
[4] C. W. Siders, A. Cavalleri, K. Sodolowski-Tinten, C. Toth, T. Guo, M. Kammler, M. Horn von Hoegen, K. R. Wilson, D. von der Linde, and C. P. J. Barty, Detection of nonthermal melting by ultrafast x-ray diffraction, Science 286, 1340 (1999).

[5] A. Rousse, C. Rischel, S. Fourmaux, I. Uschmann, S. Sebban, G. Grillon, P. Balcou, E. Forster, J. P. Geindre, P. Audebert, J. C. Gauthier, and D. Hulin, Non-thermal melting in semiconductors measured at femtosecond resolution, Nature (London) 410, 65 (2001). 
[6] K. Sokolowski-Tinten, C. Blome, C. Dietrich, A. Tarasevitch, M. H. von Hoegen, D. von der Linde, A. Cavalleri, J. Squier, and M. Kammler, Femtosecond X-Ray Measurement of Ultrafast Melting and Large Acoustic Transients, Phys. Rev. Lett. 87, 225701 (2001).

[7] T. Garl, E. G. Gamaly, D. Boschetto, A. V. Rode, B. LutherDavies, and A. Rousse, Birth and decay of coherent optical phonons in femtosecond-laser-excited bismuth, Phys. Rev. B 78, 134302 (2008).

[8] P. L. Silvestrelli, A. Alavi, M. Parrinello, and D. Frenkel, Ab Initio Molecular Dynamics Simulation of Laser Melting of Silison, Phys. Rev. Lett. 77, 3149 (1996).

[9] N. Medvedev, Z. Li, and B. Ziaja, Thermal and nonthermal melting of silicon under femtosceond x-ray radiation, Phys. Rev. B 91, 054113 (2015).

[10] P. Beaud, S. L. Johnson, E. Vorobeva, U. Staub, R. A. DeSouza, C. J. Milne, Q. X. Jia, and G. Ingold, Ultrafast Structural Phase Transition Driven by Photoinduced Melting of Charge and Orbital Order, Phys. Rev. Lett. 103, 155702 (2009).

[11] J. Kanasaki, E. Inami, K. Tanimura, H. Ohnishi, and K. Nasu, Formation of $s p^{3}$-Bonded Carbon Nanostructures by Femtosecond Laser Excitation of Graphite, Phys. Rev. Lett. 102, 087402 (2009).

[12] C. Z. Wang, K. M. Ho, M. D. Shirk, and P. A. Molian, LaserInduced Graphitization on a Diamond (111) Surface, Phys. Rev. Lett. 85, 4092 (2000).

[13] H. O. Jeschke, M. E. Garcia, and K. H. Bennemann, Microsocopic analysis of the laser-induced femtosecond graphitization of diamond, Phys. Rev. B 60, R3701 (1999).

[14] R. Nuske, A. Jurgilaitis, H. Enquist, M. Harb, Y. Fang, U. Hakanson, and J. Larsson, Transforming graphites to nanoscale diamonds by a femtosecond laser pulse, Appl. Phys. Lett. 100, 043102 (2012).

[15] N. Medvedev, V. Tkachenko, and B. Ziaja, Modelling of nonthermal solid-to-solid phase transition in diamond irradiated with femtosecond x-ray fel pulse, Contrib. Plasma Phys. 55, 12 (2015).

[16] E. S. Zijlstra, N. Huntemann, and M. E. Garcia, Laser-induced solid-solid phase transition in as under pressure: a theoretical prediction, New J. Phys. 10, 033010 (2008).

[17] Y. Giret, S. L. Daraszewicz, D. M. Duffy, A. L. Shluger, and K. Tanimura, Nonthermal solid-to-solid phase transitions in tungsten, Phys. Rev. B 90, 094103 (2014).

[18] S. T. Murphy, S. L. Daraszewicz, Y. Giret, M. Watkins, A. L. Shluger, K. Tanimura, and D. M. Duffy, Dynamical simulations of electronically induced solis-solid phase transitions in tungsten, Phys. Rev. B 92, 134110 (2015).

[19] A. P. Horsfield, M. Finnis, M. Foulkes, J. LePage, D. Mason, C. Rave, A. P. Sutton, D. R. Bowler, A. J. Fisher, R. Miranda, L. Stella, A. M. Stoneham, D. Dundas, E. McEniry, T. N. Todorov, and C. G. Sanchez, Correlated electron-ion dynamics in metallic systems, Comput. Mater. Sci. 44, 16 (2008).

[20] J. C. Tully and R. K. Preston, Trajectory surface hopping approach to nonadiabatic molecular collisions: The reaction of $\mathrm{H}^{+}$with $\mathrm{D}_{2}$, J. Chem. Phys. 55, 562 (1971).

[21] D. S. Scholl and J. C. Tully, A generalised surface hopping method, J. Chem. Phys. 109, 7702 (1998).

[22] D. S. Ivanov and L. V. Zhigilei, Combined atomistic-continuum modeling of short-pulse laser melting and disintegration of metal films, Phys. Rev. B 68, 064114 (2003).
[23] S. L. Daraszewicz, Y. Giret, N. Naruse, Y. Murooka, J. Yang, D. M. Duffy, A. L. Shluger, and K. Tanimura, Structural dynamics of laser-irradiated gold nanofilms, Phys. Rev. B 88, 184101 (2013).

[24] Z. Lin and L. V. Zhigilei, Time-resolved diffraction profiles and atomic dynamics in short-pulse laser-induced structural transformations: Molecular dynamics study, Phys. Rev. B 73, 184113 (2006).

[25] Z. Lin, E. Leveugle, E. M. Bringa, and L. V. Zhigilei, Molecular dynamics simulation of laser melting of nanocrystalline $\mathrm{Au}$, J. Phys. Chem. C 114, 5686 (2010).

[26] C. Wu, M. S. Christensen, J.-M. Savolainen, P. Balling, and L. V. Zhigilei, Generation of subsurface voids and a nanocrystalline surface layer in femtosecond laser irradiation of a single-crystal Ag target, Phys. Rev. B 91, 035413 (2015).

[27] D. M. Duffy and A. M. Rutherford, Including the effects of electronic stopping and electron-ion interactions in radiation damage simulations, J. Phys.: Condens. Matter 19, 016207 (2007).

[28] A. M. Rutherford and D. M. Duffy, The effect of electron-ion interactions on radiation damage simulations, J. Phys.: Condens. Matter 19, 496201 (2007).

[29] E. Zarkaoula, S. L. Daraszewicz, D. M. Duffy, M. A. Seaton, I. Todorov, K. Nordlund, M. T. Dove, and K. Trachenko, Electronic effects in high-energy radiation damage in iron, J. Phys.: Condens. Matter 26, 085401 (2014).

[30] S. Khakshouri, D. Alfe, and D. M. Duffy, Development of an electron-temperature-dependent interatomic potential for molecular dynamics simulation of tungsten under electronic excitation, Phys. Rev. B 78, 224304 (2008).

[31] L. Shokeen and P. K. Schelling, An empirical potential for silicon under conditions of strong electronic excitation, Appl. Phys. Lett. 97, 151907 (2010).

[32] L. Shokeen and P. K. Schelling, Thermodynamics and kinetics of silicon under conditions of strong electronic excitation, J. Appl. Phys. 109, 073503 (2011).

[33] L. Shokeen and P. K. Schelling, Role of electronic-excitation effects in the melting and ablation of laser excited silicon, Comput. Mater. Sci. 67, 316 (2013).

[34] G. E. Norman, S. V. Starikov, and V. V. Stegailov, Atomistic simulation of laser ablation of gold: Effect of pressure relaxation, J. Exp. Theor. Phys. 114, 792 (2012).

[35] Y. Rosandi, F. C. Kabeer, Y. Cherednikov, E. S. Zijlstra, M. E. Garcia, N. A. Inogamov, and H. M. Urbassek, Melting of Al induced by laser excitation of 2p holes, Mater. Res. Lett. 3, 149 (2015).

[36] S. I. Anisimov, B. L. Kapeliovich, and T. L. Perel'man, Electron emission from metal surfaces exposed to ultrashort laser pulses, Sov. Phys. JETP 39, 375 (1974).

[37] Y. Giret, A. Gelle, and B. Arnaud, Entropy Driven Atomic Motion in Laser-Excited Bismuth, Phys. Rev. Lett. 106, 155503 (2011).

[38] S. L. Daraszewicz, Y. Giret, H. Tanimura, D. M. Duffy, A. L. Shluger, and K. Tanimura, Determination of the electronphonon coupling constant in tungsten, Appl. Phys. Lett. 105, 023112 (2014).

[39] Z. Lin, L. V. Zhigilei, and V. Celli, Electron-phonon coupling and electron heat capacities of metals under conditions of strong 
electron-phonon nonequilibrium, Phys. Rev. B 77, 075133 (2008).

[40] X. Gonze, B. Amadon, P.-M. Anglade, J.-M. Beuken, F. Bottin, P. Boulanger, F. Bruneval, D. Caliste, R. Caracas, and M. E. A. Cote, Abinit: First-principles approach to material and nanosystem properties, Comput. Phys. Commun. 180, 2582 (2009).

[41] X. Gonze, G.-M. Rignanese, M. Verstraete, J.-M. Beuken, Y. Pouillon, R. Caracas, F. Jollet, M. Torrent, G. Zerah, M. Mikami, P. Ghosez, M. Veithen, J.-Y. Raty, V. Olevano, F. Bruneval, L. Reining, R. Godby, G. Onida, D. R. Hamann, and D. C. Allan, A brief introduction to the abinit software package, Z. Kristallogr. 220, 558 (2005).

[42] E. Gamaly, Femtosecond Laser-Matter Interaction: Theory, Experiments and Applications (CRC Press, Boca Raton, FL, 2011).

[43] E. G. Gamaly and A. V. Rode, Electron-phonon energy relaxation in bismuth excited by ultrashort laser pulse: temperature and fluence dependence, Appl. Phys. A 110, 529 (2013).

[44] P. B. Allen, Misbehavior of metals, Nature (London) 405, 1007 (2000).

[45] K. Eidmann, J. Meyer-ter-Vehn, T. Schlegel, and S. Huller, Hydrodynamic simulation of subpicosecond laser interaction with solid density matter, Phys. Rev. E 62, 1202 (2000).

[46] G. V. Sin'ko, N. A. Smirnov, A. A. Ovechkin, P. R. Levashov, and K. V. Khishchenko, Thermodynamic functions of the heated electron subsystem in the field of cold nuclei, High Ener. Dens. Phys. 9, 309 (2013).

[47] E. Bevillon, J. P. Colombier, V. Recoules, and R. Stoian, Free electron propereties of metals under ultrafast laser-induced electron-phonon non-equilibriums: a first principles study, Phys. Rev. B 89, 115117 (2014).

[48] E. Bevillon, J. P. Colombier, V. Recoules, and R. Stoian, First principles calculations of heat capacities of ultrafast laser-excited electrons in metals, Appl. Surf. Sci. 336, 79 (2015).

[49] J. F. Lutsko, D. Wolf, S. R. Phillpot, and S. Yip, Molecular dynamics study of lattice-defect-nucleated melting in metals using an embedded-atom-method potential, Phys. Rev. B 40, 2841 (1989).

[50] I. T. Todorov, W. Smith, K. Trachenko, and M. T. Dove, Dlpoly3: new dimensions in molecular dynamics simulations via massive parallelism, J. Mater. Chem. 16, 1911 (2006).

[51] D. S. Ivanov and L. V. Zhigilei, Kinetic Limit of Heterogeneous Melting in Metals, Phys. Rev. Lett. 98, 195701 (2007).

[52] D. S. Ivanov and L. V. Zhigilei, Effect of Pressure on the Mechanisms of Short-Pulse Laser Melting, Phys. Rev. Lett. 91, 105701 (2003).

[53] A. K. Upadhyay and H. M. Urbassek, Response of ultrathin metal films to ultrafast laser irradiation: A comparative molecular-dynamics study, J. Phys.: Confer. Series 59, 68 (2007).

[54] J. Chen, W.-K. Chen, J. Tang, and P. M. Rentzepis, Timeresolved structural dynamics of thin metal films heated with femtosecond optical pulses, Proc. Nat. Acad. Sci. USA 108, 18887 (2011).

[55] C. Laulhe, M. Cammarata, M. Servol, R. J. Dwayne-Miller, M. Hada, and S. Ravy, Impact of laser on bismuth thin-films, Eur. Phys. J. Special Topics 222, 1277 (2013).

[56] S. Feng and X. Cheng, First-principles investigation on metal tantalum under conditions of electronic excitation, Comput. Mater. Sci. 50, 3110 (2011).

[57] H. J. Fecht and W. L. Johnson, Entropy and enthalpy catastrophe as a stability limit for crystalline material, Nature (London) 334, 50 (1988).

[58] T. Zier, E. S. Zijlstra, and M. E. Garcia, Silicon before the bonds break, Appl. Phys. A 117, 1 (2014). 